THURSDAY, FEBRUARY 8, 1906.

\section{A NEW INTEGRAL CALCULUS.}

Integral Calculus for Beginners. By Alfred Lodge, M.A. Pp. xiii +203. (London: George Bell and Sons, 1905.) Price $4 s .6 d$.

"THIS is a sequel to the author's 'Differential 1 Calculus, and is intended for students of physics and mechanics who require a good working knowledge of integration and its more simple applications." Such is the claim put forward by Prof. Lodge in his preface. We naturally expect a bouk in which simple useful applications figure more prominently than lengthy theoretical investigations, and in this we are not disappointed. Moreover, a number of interesting features strike us as being particularly good, although a few others are capable of improvement.

The first of the good features is the insertion of the integration constant $\mathrm{C}$ in the elementary workedout examples on integration. Its omission frequently leads a beginner astray. Another feature possessing many advantages is that the chapter on rational fractions is reduced to a minimum. The process of integrating a rational fraction with a denominator of high degree is not often required in actual practice. Moreover, the graph of such a fraction has infinite branches corresponding to the real factors of the denominator, so that unless the areas of these infinite branches are carefully discussed, by introducing the notion of the "principal value" of a definite integral, the result only enables us to find the areas of limited portions of the curves, for which approximate methods of quadrature would in many cases suffice. On the other hand, the mode of introducing the connection between integration and summation-a point on which Prof. Lodge rightly lays special stress $(\S \S 2,43,48)$--will probably be regarded by most readers as not so satisfactory as it might be. Thus, to go no further, a relation on p. 4 is stated to be true to the first order which on p. 62 is shown to contain an error of the first order. This is the greater pity as the investigation of $\S 48$ would, with the addition of a couple of lines, contain all that is necessary for a rigorous graphical proof, far shorter than that given in $\S 2$; we hope this point will receive attention in future editions.

In reading the sections dealing with Simpson's rule and its modifications, one is surprised at the conservatism that prevails in the retention of a formula in which odd and even ordinates have unequal weight -a conservatism quite independent of the present book. When it is recollected that cutting off the first and last strips of a curvilinear area reverses the weights of the ordinates it will be seen easily that the trapezoidal rule, modified by suitable corrections for the two ends, may be made to give results quite as accurate as those of Simpson's rule.

No. 1893 , voL. 73]
Under applications of the calculus, we find areas, centres of mass, volumes and surfaces of revolution, and moments of inertia with especial reference to plane areas and their centres of pressure. The sections on differential equations contain what has for some time past been regarded as a standard elementary course on the subject, namely, the simpler equations of the first order and linear equations with constant coefficients. The study of the first integral of the equation $d^{2} y / d x^{2}=f(y)$ in connection with its kinematic interpretation, and the discussion of small oscillations in connection with the equation of harmonic motion, are good features. Finally, the chapter on the Gamma function has given Prof. Lodge an opportunity of saying something he wanted to say, and of saying it in his own way instead of cutting or drying it down to the requirements of a syllabus. It contains an interesting discussion of the extension of the conception of a factorial to negative and fractional arguments. It is much to be hoped that this chapter will encourage other writers of text-books to launch out into something new and original. This might to some extent help to save English mathematical teaching from sinking down to a uniform dead level of mediocrity, reminding one of an open, barren veldt, in which all the smaller hills have been levelled down by the steam-roller of the examination and the syllabus, and all high eminences have crumbled to the ground as a result of the starvation salaries paid to really competent mathematical teachers.

G. H. BRYAN.

[Since the above was written I have had some correspondence with Prot. Lodge quite independently of the present review. The treatment of the summation of infinitesimals contemplated by him in the articles criticised above may be stated more clearly somewhat as follows:-Let $y=f(x)$, and let $x$ increase from $a$ to $b$ by a series of increments $d x$. Then if $d y$ denote the corresponding increment of $y$, the sum of the increments $d y$ is exactly equal to $f(b)-f(a)$. Moreover, the "exact differential" $d y$ becomes equal to the "differential product" $f^{\prime}(x) d x$ when $d x$ is infinitesimal, and under this condition we may put $f(b)-f(a)$, equal also to the sum of the differential products $f^{\prime}(x) d x$. Also in all practical applications Prof. Lodge contends that what we really want is the sum of the exact differentials $d y$ rather than the sum of the corresponding differential products $f^{\prime}(x) d x$. This contention I believe to be correct, and if Prof. Lodge can re-write the articles once more -for he says that he has already repeatedly re-written them-and make it more clear that he is not merely giving an inaccurate reproduction of Todhunter's rigorous proof, but something quite different, his treatment may be made one of the many valuable features of his book. The method interests me greatly, and appears to be of sufficient general interest to justify the present explanatory note.G. H. B.] 